\title{
Robust Estimation of Curvature along Digital Contours with Global Optimization
}

\author{
Bertrand Kerautret ${ }^{1}$ and Jacques-Olivier Lachaud ${ }^{2, \star}$ \\ ${ }^{1}$ LORIA, Nancy- Campus Scientifique \\ 54506 Vandœuvre -lès-Nancy Cedex \\ kerautre@loria.fr \\ ${ }^{2}$ LAMA, University of Savoie \\ 73376 Le Bourget du Lac \\ jacques-olivier.lachaud@univ-savoie.fr
}

\begin{abstract}
In this paper we introduce a new curvature estimator based on global optimisation. This method called Global Min-Curvature exploits the geometric properties of digital contours by using local bounds on tangent directions defined by the maximal digital straight segments. The estimator is adapted to noisy contours by replacing maximal segments with maximal blurred digital straight segments. Experimentations on perfect and damaged digital contours are performed and in both cases, comparisons with other existing methods are presented.
\end{abstract}

\section{Introduction}

Estimating geometric characteristics of digital shapes is an essential step in many image analysis and pattern recognition applications. We focus here on the geometry of digital 4-connected contours. These contours arise naturally as the inter-pixel boundary of digital regions in images. We present here a new method for estimating the curvature at any point of such digital contours, i.e. we estimate the curvature field of the contour. We are interested here by the quantitative estimation of the curvature field and not only the detection of dominant or inflexion points, as opposed to many methods proposed in the pattern recognition community (see [1]). Note however that detecting these points is a natural byproduct of curvature computation, provided curvature estimations are stable enough. Furthermore digital contours are rarely perfect digitizations of regular shapes, as may be seen on Fig.1 In order to be useful, curvature estimation techniques should thus be able to take into account local perturbations, provided the digital contour holds more significant information than noise.

To estimate a geometric characteristic from a digital contour, it is necessary to suppose that there is an underlying real shape, although its geometry is generally unknown. A "good" estimator aims at approaching at best the corresponding geometric characteristic of this real shape. It is however difficult if not impossible to compare objectively the respective accuracy of several estimators, since

\footnotetext{
* This work was partially funded by the ANR project GeoDIB. Bertrand Kerautret was partially funded by a BQR project of Nancy University.
} 

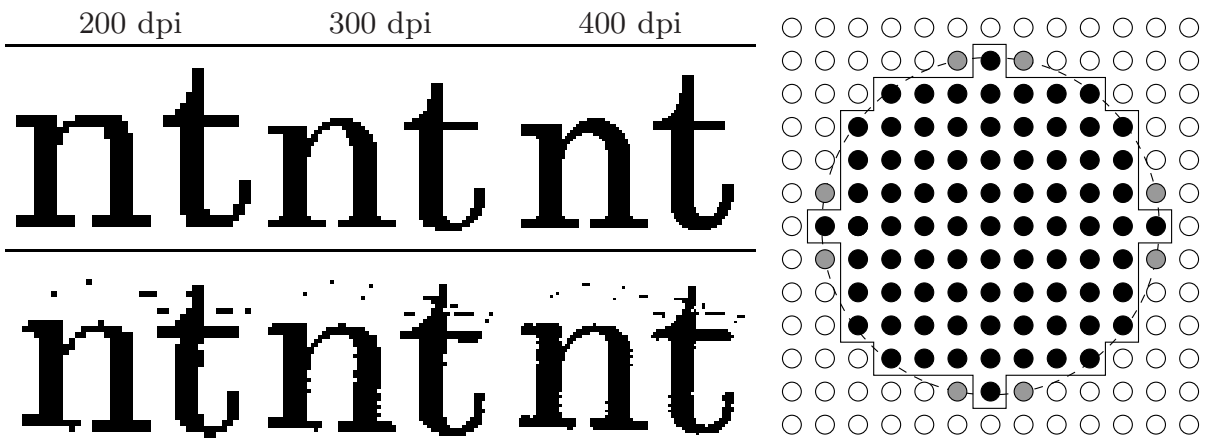

Fig. 1. Left. Perfect versus noisy digital contours. Top row: the letters "nt" written in a roman font family of size $14 \mathrm{pt}$ are digitized at several increasing resolution. Bottom row: the same text has been printed at 600dpi on a laser printer and then scanned at the corresponding resolution. The digital contour of both letters has been damaged in the process and presents some irregularities which are very visible on straight parts. Pepper and salt noise is also visible. Furthermore, these phenomena are visible at each resolution. Right. Digital object $O$ depicted with black disks $\bullet$. Its shape of reference is the disk sketched with dotted lines, whose radius is constrained by the gray disks. The perfect curvature map is thus $\kappa(t)=1 / \sqrt{5^{2}+1}$.

for a given digital contour there exists infinitely many shapes with the same digitization. This problem is even greater here, since infinitesimal perturbations in the input shape may induce huge variations in the curvature field.

Before going further, we introduce some notations and definitions (mainly taken from [2]). Let $\operatorname{Dig}_{h}$ be the Gauss digitization process of step $h$ (i.e. the intersection of $\mathbb{Z}^{2}$ with the magnification of the shape by $\left.1 / h\right)$. Let $\mathbb{F}$ be a family of shapes in $\mathbb{R}^{2}$ with appropriate properties 1 . Let $G$ be some geometric feature defined for any shape $X$ of $\mathbb{F}$. A discrete geometric estimator $E_{G}$ of $G$ is a map that associates to a digitization $\operatorname{Dig}_{h}(X)$ an estimation of $G(X)$. Properties of geometric estimators are classically defined for global shape features like area or perimeter (see [2]). We adapt this definition to local geometric features like tangent or curvature as follows (other definitions may be found 34]). Let $s$ be the arc length parameterization of the topological boundary $\partial X$ of $X$, and let $t$ be $s$ divided by the length of $\partial X$. Here the feature $G$ is the curvature field, which may be then represented as a map $\kappa: t \in[0,1] \mapsto \kappa(t) \in \mathbb{R}$. Our purpose is therefore to build a function $E_{\kappa}$ which approaches $\kappa$ at best as possible, for instance in the $L^{2}([0,1])$ sense.

As far as we know, the only property of geometric estimators that can objectively be compared is the multigrid convergence, which indicates that the finer the digitization step, the better is the estimation. Generally speaking, the estimator $E_{G}$ is multigrid convergent toward $G$ for the family $\mathbb{F}$ iff, for any shape $X \in \mathbb{F}$, there exists some $h_{X}>0$ for which

\footnotetext{
${ }^{1}$ We take here the family of connected compact shapes whose boundary is rectifiable and whose curvature map is in $L^{2}$. This avoids fractal-like shapes and the curvature, while not compulsory defined everywhere, is therefore square integrable.
} 


$$
\forall h, 0<h<h_{X},\left\|E_{G}\left(\operatorname{Dig}_{h}(X)\right)-G(X)\right\| \leq \tau(h),
$$

where $\tau: \mathbb{R}^{+} \rightarrow \mathbb{R}^{+*}$ has limit value 0 at $h=0$. This function defines the speed of convergence of $E_{G}$ toward $G$. This property seems appealing for comparing geometric estimators, since a good speed of convergence guarantees a good estimation at a high enough scale.

The multigrid convergence of several geometric estimators has been studied in the literature: area [5], moments [6], perimeter [7/8/9], tangents [10]11/3|12]. For the curvature field, the multigrid convergence is not yet achieved 101314, although a very recent approach based on global filtering by a carefully chosen binomial kernel seems promising [15]. The multigrid convergence may nevertheless be criticized on the following points: (i) these estimators are guaranteed to be precise only at very fine resolution; (ii) this property has meaning only for perfect shape digitizations: it is no more valid when the input data has been more or less slightly — damaged.

We propose a new objective criterion for curvature estimation which is valid also at coarse resolution (addressing criticism (i)). Furthermore, we show how to compute a numerical approximation of the optimal solution for this criterion and then how to adapt this algorithm to corrupted or noisy data (addressing criticism (ii)). More precisely, a good objective criterion should take into account not only one real shape but all the real shapes that have the input digital contour as digitized boundary. Of course, not all those real shapes should have the same probability to be the true shape of interest. For instance, a very classical tool in image analysis are the deformable models of Kass et al. [16] or the geodesic active contours which considers shapes with short perimeters as more likely than shapes with winding contours [17. These methods consider shapes with smooth contours as more preferable than shapes with many points of high curvature.

Following this analogy, given a digital object $O$ (a non-empty finite subset of $\mathbb{Z}^{2}$ ), we define the shape of reference $R_{O, h}$ to $O$ at grid step $h$ as the shape of $\mathbb{F}$ which minimizes its squared curvature along its boundary and such that $\operatorname{Dig}_{h}\left(R_{O, h}\right)=O$. An illustration is given in Fig.1] right. We therefore define

Definition 1. Given a curvature estimator $E_{\kappa}$, its min-curvature criterion relative to object $O$ and step $h$ is the positive quantity $\left\|E_{\kappa}-\kappa_{O, h}\right\|$, where $\kappa_{O, h}$ is the curvature map of $R_{O, h}$ and $\|\cdot\|$ is the $L^{2}$-norm.

A good curvature estimator should therefore have a low min-curvature criterion for a large family of shapes. One may notice that a similar criterion is implicitly used for perimeter estimation by Sloboda et al. 8]: their perimeter estimator is defined as the perimeter of the polygon which has the same digitization as the input digital object and which minimizes its perimeter. In other terms, their shape of reference is the one that minimizes $\int_{\partial X} 1$. Their perimeter estimator has good properties at low scale but is also multigrid convergent with speed $O(h)$.

We present here a curvature estimator, called Global Min-Curvature estimator (GMC) whose min-curvature criterion is numerically zero for the family of shapes $\mathbb{F}$, i.e. it is the best possible curvature estimator for this criterion. Our method 
exploits the specific geometric properties of digital contours. The maximal digital straight segments of the input digital contour are used to define local bounds on the tangent directions (Section 2). These bounds are casted into the space of tangent directions. The curvature of the reference shape $O$ is then computed by optimization in this tangent space (GMC estimator (Section 3)). In Section 4, we adapt our estimator to noisy or damaged digital contours by replacing maximal segments with maximal blurred digital straight segments. Section 5 validates our curvature estimator with several experiments on perfect or damaged digital contours, and with some comparisons with other curvature estimators.

\section{Tangential Cover and Tangent Space}

We assume that the input data is the inter-pixel boundary of some digital objects, that we will call later on a digital contour. It is given as a 4-connected closed path $C$ in the digital plane, whose discrete points $C_{i}$ are numbered consecutively. A sequence of connected points of $C$ going increasingly from $C_{i}$ to $C_{j}$ is conveniently denoted by $C_{i, j}$.

Such a sequence is a digital straight segment iff its points are included in some standard digital straight line, i.e. $\exists(a, b, \mu) \in \mathbb{Z}^{3}, \forall k, i \leq k \leq j, \mu \leq a x_{C_{k}}-b y_{C_{k}}<$ $\mu+|a|+|b|$. The standard line with smallest $|a|$ and containing the sequence, defines the characteristics $(a, b, \mu)$ of the digital straight segment. In particular, the slope of the segment is $a / b$. Let us now denote by $S(i, j)$ the predicate " $C_{i, j}$ is a digital straight segment". A maximal segment of $C$ is a sequence $C_{i, j}$ such that $S(i, j) \wedge \neg S(i, j+1) \wedge \neg S(i-1, j)$. The maximal segments are thus the inextensible digital straight segments of $C$. Together, they constitute the tangential cover of $C$, as illustrated on Fig.2, left.

The tangential cover of a digital contour can be efficiently computed in linear time with respect to its number of points 1812. The directions of maximal segments may be used to estimate the tangent direction of the underlying shape [11. Here we also make use of the direction of maximal segments, but to estimate locally the geometries of all possible underlying shapes. We proceed as follows.

1. Each maximal segment tells us some information on the local geometry of the underlying continuous shape (Fig.2, right). In particular, the direction of maximal segment gives bounds on the possible tangent directions of the continuous shape. Although this can be done in several ways, we choose to estimate these bounds from the leaning points. If $M$ and $M^{\prime}$ are the two furthest upper or furthest lower leaning points, then the minimal slope (resp. maximal slope) is chosen as the slope of the segment joining $M$ to $M^{\prime}+$ $(0,-1)$ (resp. $M$ to $\left.M^{\prime}+(0,1)\right)$. For a maximal segment of characteristics $(a, b)$ and with only two upper leaning points and at most two lower leaning points, its extremal slopes are thus $\frac{a}{b} \pm \frac{1}{b}$.

2. We represent a closed $C^{1}$-curve $\mathcal{C}$ parameterized by its arc length $s$ as a function graph which maps $s$ to the tangent direction at $\mathcal{C}(s)$. The domain is $[0, L[, L$ being the curve length and the range is $[0,2 \pi$. Such a representation, that we call hereafter tangent space, defines the closed curve geometry up to a translation. 


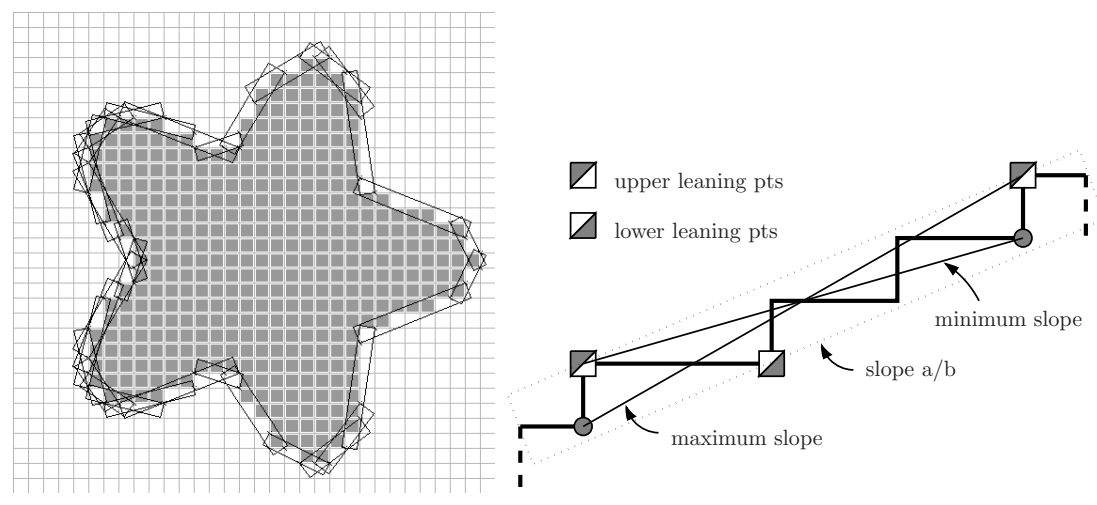

Fig. 2. Left: tangential cover of the boundary of a digitized shape, where each maximal segment is drawn as a black bounding box aligned with its slope. Right: slope of a maximal segment and estimation of maximal and minimal slopes with leaning points.

3. We fix $C_{0}$ as the starting point of the arc length parameterization. Given a digital length estimator $E_{L}$, we can estimate the arc length $s_{i}$ of any point $C_{i}$ as $E_{L}\left(C_{0} C_{i}\right)$. For each maximal segment $C_{i, j}$, we then draw in the tangent space an axis aligned box spanning abscissas $s_{i}$ to $s_{j}$ and whose ordinates are the inverse tangent of the extremal slopes (Fig. 3, left).

4. Although infinitely many shapes have the same digitization as the input digital object, the boundary of shapes with smooth enough tangents should have a tangent space representation that stays within the boxes defined above. On first approximation, the boundary contour is indeed locally a straight line.

\section{Curvature Computation by Optimization}

We extract the shape of reference to the input digital object $O$ from the tangent space representation. Indeed, if the shape boundary $\mathcal{C}$ is smooth enough, its geometry is entirely defined by the mapping $\theta_{\mathcal{C}}$ which associates to an arc length $s$ the direction of the tangent at point $\mathcal{C}(s)\left(\theta_{\mathcal{C}}=\angle\left(0 x, \mathcal{C}^{\prime}\right)\right)$. Since the curvature is the derivative of the tangent direction, the integral $J[\mathcal{C}]$ along $\mathcal{C}$ of its squared curvature is then

$$
J[\mathcal{C}]=\int_{\mathcal{C}} \kappa^{2}=\int_{0}^{L} \kappa^{2}(s) d s=\int_{0}^{L}\left(\frac{d \theta_{\mathcal{C}}}{d s}\right)^{2} d s .
$$

The shape of reference to $O$ is the shape in $\mathbb{F}$ of boundary $\mathcal{C}$ which minimizes $J[\mathcal{C}]$ and which is digitized as $O$. From the preceding section, the tangent space representation of $\mathcal{C}$ should stay within the bounds given by the maximal segments. Let us now denote $\left(i_{l}\right)$ the increasing sequence of indices of the digital points that are starting or ending point of a maximal segment. To each point $C_{i_{l}}$, we 

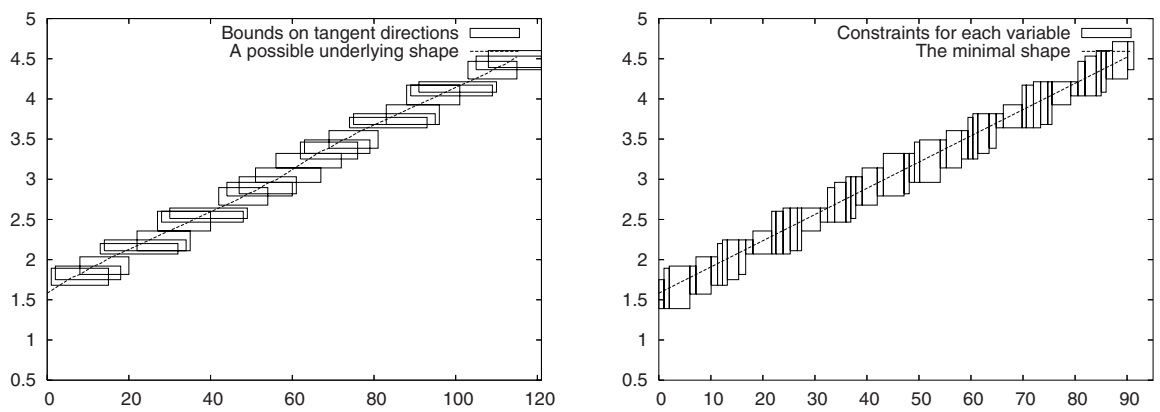

Fig. 3. The shape of interest is a digitized disk of radius 30.5. Left: bounds given by each maximal segment on the possible local tangent direction (abscissa: index of the contour point, ordinate: angle wrt axis $x$ ). A possible underlying space should have its tangent space representation staying within these boxes. Right: Each variable has a possible range given by its vertical line. The shape that minimizes its squared curvature is represented by the dashed line. The obtained curvature field is constant and corresponds to the one of a disk of radius 30.5 .

associate the smallest possible tangent direction $a_{l}$ as the smallest bound given by the maximal segments strictly containing $C_{i_{l}}$. The largest possible tangent direction $b_{l}$ is defined symmetrically (see. Fig.33). Finally let $t_{l}$ be the tangent direction of the shape of reference at its point of arc length $s_{i_{l}}$.

The digitization constraint imposes $\forall l, a_{l} \leq t_{l} \leq b_{l}$. We look for a shape of reference with smooth enough boundary (which we will precise later). Its mapping $\theta_{\mathcal{C}}$ is therefore continuous and interpolates points $\left(s_{i_{l}}, t_{l}\right)$. Looking now at an arbitrary portion $\left[s_{i_{l}}, s_{i_{l+1}}\right]$ of the curve, standard variation calculus on Equ (2) immediately gives the necessary condition $2 \frac{d}{d s} \frac{d \theta_{\mathcal{C}}}{d s}=0$, otherwise said the straight segment $\theta_{\mathcal{C}}(s)=t_{l}+\frac{t_{l+1}-t_{l}}{s_{i_{l+1}}-s_{i_{l}}}\left(s-s_{i_{l}}\right)$. A straight segment in the tangent space is a circular arc in the plane. It is straightforward to check that this segment stays within the bounds defined by the maximal segments.

We have therefore found the optimal boundary of the shape of reference, if the family $\mathbb{F}$ of shapes is composed of shapes with $C^{0}$ and piecewise $C^{1}$ continuity of tangent direction. It must be found in the family $\mathcal{C}\left[\ldots, t_{l}, \ldots\right]$ whose tangent directions are piecewise linear functions. Curvature estimation of reference shape to $O$ is thus reduced to

Find $\left(t_{l}\right)_{l}$, which minimizes $J\left[\mathcal{C}\left[\ldots, t_{l}, \ldots\right]\right]=\sum_{l}\left(\frac{t_{l+1}-t_{l}}{s_{i_{l+1}}-s_{i_{l}}}\right)^{2}\left(s_{i_{l+1}}-s_{i_{l}}\right)$, subject to $\quad \forall l, a_{l} \leq t_{l} \leq b_{l}$.

We use classical iterative numerical techniques to solve this optimization problem, simply following $\frac{\partial J}{\partial t_{l}}$ for each variable $t_{l}$ while staying in the given interval. Geometrically, each variable $t_{l}$ is moved toward the straight segment joining $\left(s_{i_{l-1}}, t_{l-1}\right)$ to $\left(s_{i_{l+1}}, t_{l+1}\right)$. The GMC estimator $E_{\kappa}^{\mathrm{GMC}}$ is then simply defined as the derivative of the piecewise linear function joining points $\left(s_{i_{l}}, t_{l}\right)$, rescaled by 
$h$. Being a piecewise constant function, this curvature estimator is very stable and is undefined only on a zero-measure set. From Definition [1, it is obvious that the GMC estimator is (numerically) zero.

Fig. 3. right, illustrates this computation for a digital disk. Each variable $t_{l}$ is bounded by the drawn vertical interval. The tangent direction of the shape of reference is the piecewise linear function drawn with a dashed line: it is here a straight line since there exists a continuous disk with same digitization. The GMC estimation is thus constant and is approximately the inverse of the digital disk radius. Let us finally note that the number of variables to optimize is considerably lower than the number $N$ of points, and is some $O\left(N^{\frac{2}{3}}\right)$ on shapes satisfying certain conditions (see [14 for more details). The computational cost and the iteration number needed for the optimisation are illustrated in Section 5 .

\section{Tangential Cover of Noisy Digital Contours}

If we consider noisy digital contours it seems natural to adapt the concept of tangential cover by using blurred segments. A linear time recognition algorithm of blurred segments was proposed by Debled et al. [19. This approach is based on the computation of the convex hull and on its vertical geometric width. Note that Buzer proposed a very similar approach [20]. Both of these methods assume points are added with increasing $\mathrm{x}$ coordinate in the recognition process.

In the same way of Roussillon et al. [21, we adapt the algorithm of Debled et al. [19] to avoid the restrictive hypothesis which assumes that points are added with increasing $x$ coordinate (or $y$ coordinate). The figure on the right shows an example of such a contour (light gray) with a maximal segment of width $\nu=5$ (dark gray). Despite non increasing $x$ (and y) coordinate, the resulting maximal blurred segment is well detected.

The tangent directions range $R=\left[\theta_{\min }, \theta_{\max }\right]$ associated to a maximal blurred segment can be determined by the slope of its bounding line and by its width $\nu$. More precisely, if $V\left(v_{x}, v_{y}\right)$ is the vector given by the two leaning points of the upper bounding line, the interval is defined by $R=$ $\left[\tan ^{-1}\left(\frac{v_{y}+\nu}{v_{x}}\right), \tan ^{-1}\left(\frac{v_{y}-\nu}{v_{x}}\right)\right]$. Here the value of $\nu$ was determined from the vertical width of the blurred segment convex hull. The figure on the

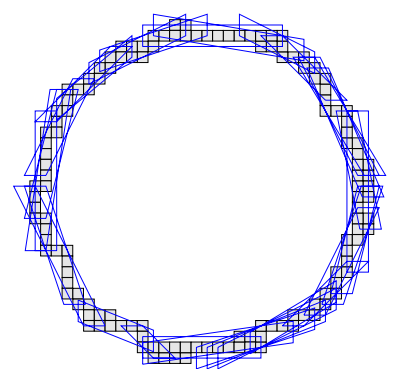
right illustrates the maximal blurred segments obtained on a noisy circle with initial radius 15. The maximal width $\nu$ of the blurred segment was set to 2 .

To compute curvature by optimization, the maximal and minimal slopes for each point need to be evaluated. It may happen that two maximal segments including the points of interest have opposite directions. Therefore, taking the minimum (resp. maximum) of the minimal (resp. maximal) slope of these maximal segments is not always coherent. So for each point we define an oriented interval associated to the minimal and maximal tangent directions: $I_{k}=\left[\theta_{\min }^{k}, \theta_{\max }^{k}\right]$. 
Then the strategy to define the global interval $I$ is to merge each interval to each other. The merging process between two intervals $I_{1}$ and $I_{2}$ can be done according to the following conditions:

(1) $I_{2}$ is included in $I_{1}: \theta_{\min }^{2}, \theta_{\max }^{2} \in\left[\theta_{\min }^{1}, \theta_{\max }^{1}\right]$ and $\theta_{\min }^{2} \in\left[\theta_{\min }^{1}, \theta_{\max }^{2}\right]$ and $\theta_{\max }^{2} \in\left[\theta_{\min }^{2}, \theta_{\max }^{1}\right]$ then the resulting interval is obviously $I_{1}$ (see for example Fig. 4 a).

(2) $I_{1}$ is included in $I_{2}$ : same case as (1) by substituting $I_{1}, I_{2}$ and $S_{1}, S_{2}$.

(3) $\theta_{\min }^{2} \in\left[\theta_{\min }^{1}, \theta_{\max }^{1}\right]$ and $\theta_{\max }^{2} \notin\left[\theta_{\min }^{1}, \theta_{\max }^{1}\right]$ then $I=\left[\theta_{\min }^{1}, \theta_{\max }^{2}\right]$.

(4) $\theta_{\max }^{2} \in\left[\theta_{\min }^{1}, \theta_{\text {max }}^{1}\right]$ and $\theta_{\min }^{2} \notin\left[\theta_{\min }^{1}, \theta_{\max }^{1}\right]$ then $I=\left[\theta_{\min }^{2}, \theta_{\max }^{1}\right]$ (Fig. $4 \mathrm{~b}$ ).

(5) $I_{1} \cup I_{2}$ is not connected: we compute the distances between the two intervals $d_{1}=\left|\theta_{\min }^{2}-\theta_{\max }^{1}\right|$ and $d_{2}=\left|\theta_{\min }^{1}-\theta_{\max }^{2}\right|$. The resulting interval is determined according to the following conditions:

- $d_{1}<d_{2}$ : the resulting interval is $I=\left[\theta_{\min }^{1}, \theta_{\max }^{2}\right]$.

- $d_{2}<d_{1}$ : the resulting interval is $I=\left[\theta_{\min }^{2}, \theta_{\max }^{1}\right]$ (see Fig.4 4 ).

(6) In all the other cases the resulting interval is set to $I=[0,2 \pi]$. Fig. 4 d shows such an example.

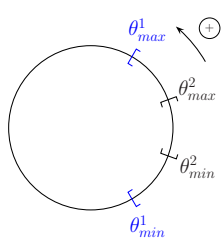

(a)

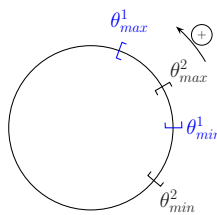

(b)

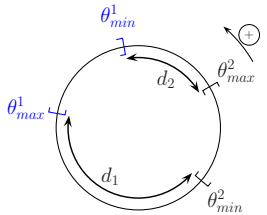

(c)

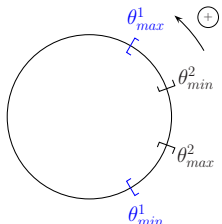

(d)

Fig. 4. Illustration of several cases which can appear in the merging process

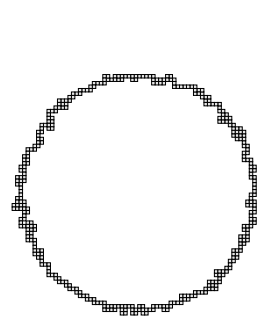

(a)

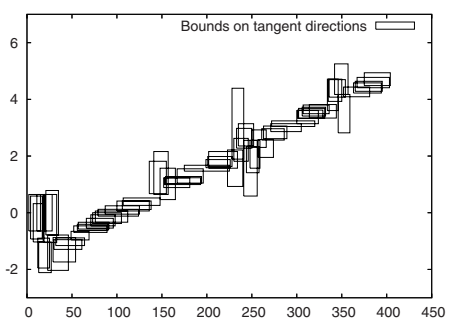

(b)

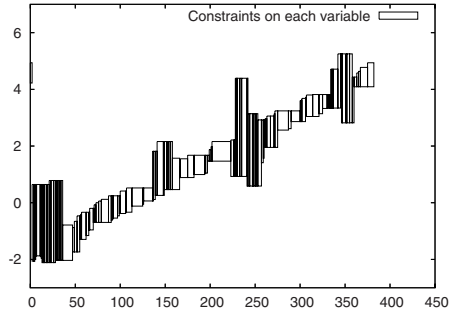

(c)

Fig. 5. Illustration of a distorted digital circle (a) with its tangent directions (b) and the constraints on each variable (c). The maximal blurred segments were obtained with width $\nu=3$.

Note that cases (1-4) appear most of the time. But on very noisy contours, the more the width $\nu$ is small, the more cases (5) and (6) appear. In such cases, the local contour geometry is not well approached by the tangential cover. Fig. 5 b 
shows the slope range of maximal blurred segments obtained from the noisy contour represented in (a). The width of the blurred segments was set to 3 . Fig. 5. shows the resulting slope range obtained after the merging process. The total number of merging configurations (1-4) was equal to 410 while configuration (5) appears 18 times in the merging process. With width $\nu=5$ the number of configurations (5) and (6) was equal to 0 . This distribution of the different cases may define a new strategy to automatically choose the width $\nu$ that is best adapted to a given noisy contour.

\section{Experimental Validation}

The GMC estimator was first experimented on a circle of radius 20 with different grid steps (Fig.7a). Even with coarse grid step, the estimator gives precise result in comparison to the method of osculating circles 2 [10] (see Fig.7b). A second experimentation was made on the flower of Fig.2, left, whose inner radius is 15 and outer radius is 25 . In the same way as the previous experimentations, results are very precise with the different grid steps and once again results are better than those obtained by the estimator based on osculating circles.

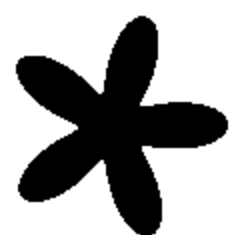

(a)

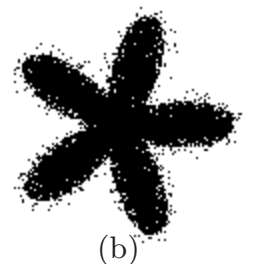

(b)

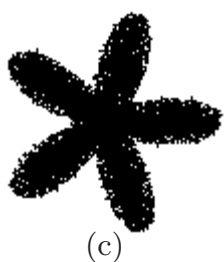

(c)

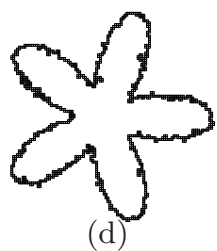

(d)

Fig. 6. Generation of noisy digital contours. Pixels from image (a) were changed according to the distance to the initial shape and according to a Gaussian distribution (image (b)). The inner component extracted from image (b) is shown in (c), and its boundary is displayed in $(\mathrm{d})$. It is a noisy version of the original flower contour.

To experiment the robustness of the curvature estimator, noisy contours were generated from synthetic objects. The main idea to obtain such contours is to compute the distance map from the initial shape boundary. From these distances we randomly change the pixel values according to a Gaussian distribution. The inner component defines the digital contour and is extracted by tracking its inter-pixel boundary. This process is illustrated in Fig.6. Note that this method of digital contour perturbation follows the local document degradation model proposed by Kanungo 22 .

The curvature estimation was experimented on several noisy shapes with different width $\nu$ of blurred segments. Fig. $7 \mathrm{f}$-h shows the curvature fields obtained on distorted disk and flower contours. The two contours were obtained from ideal smooth

${ }^{2}$ Other experimental results can be found at the following address:

http://www.loria.fr/\$ \$sim\$kerautre/dgci08/ 


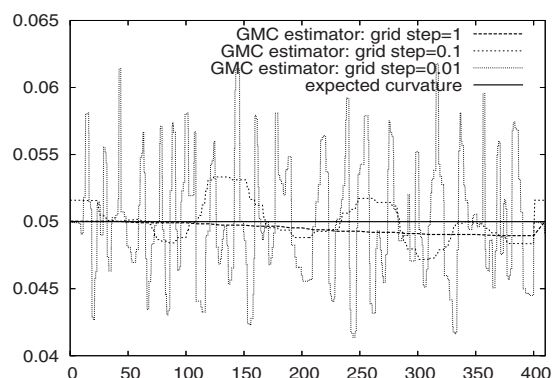

(a) Circle Radius $R=20$

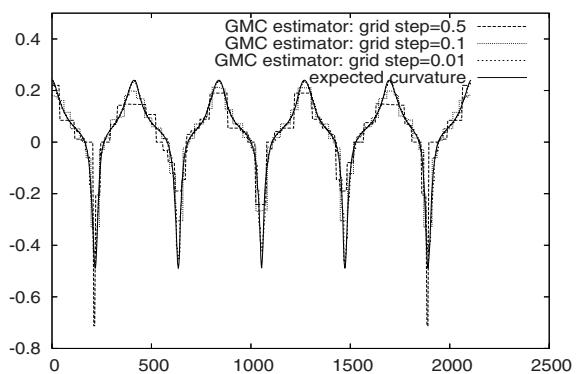

(c) Flower with 5 petals

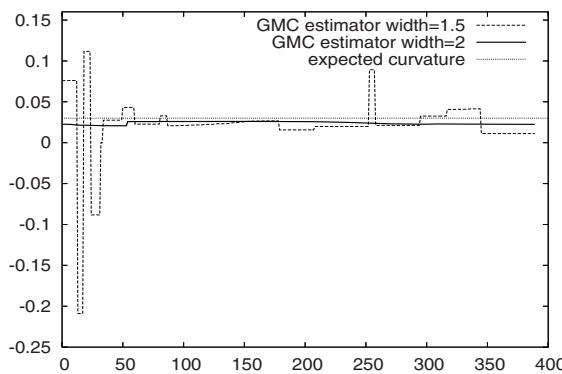

(e) "noisy" circle of Fig. 5a

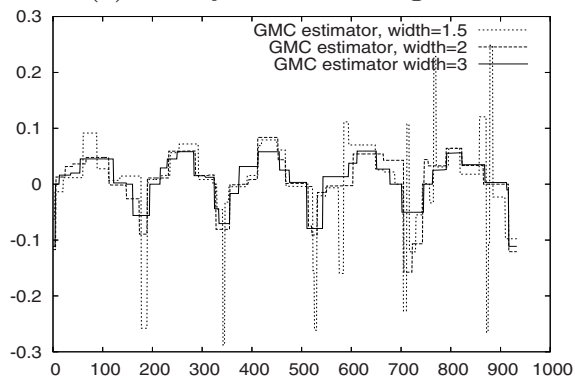

(g) "noisy" flower of Fig. 6d

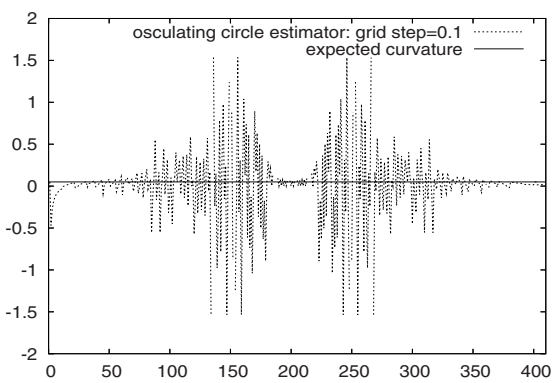

(b) Circle Radius $R=20$

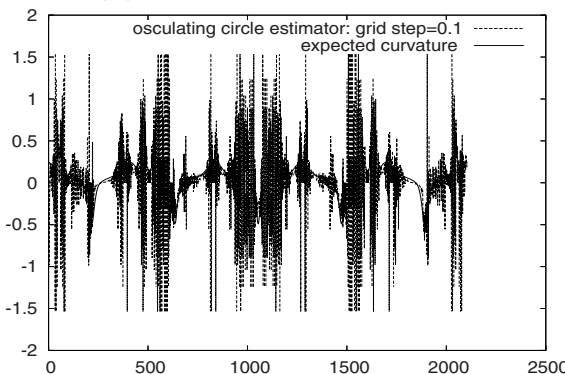

(d) Flower with 5 petals

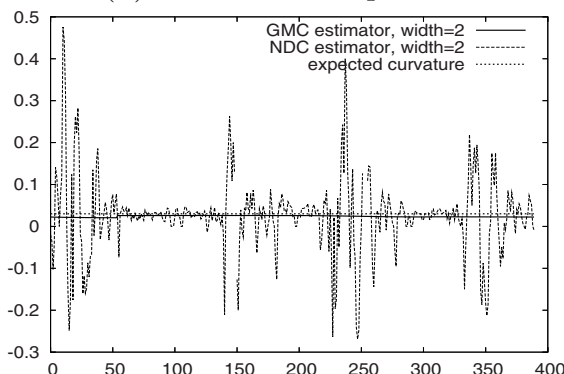

(f) "noisy" circle of Fig. 5a

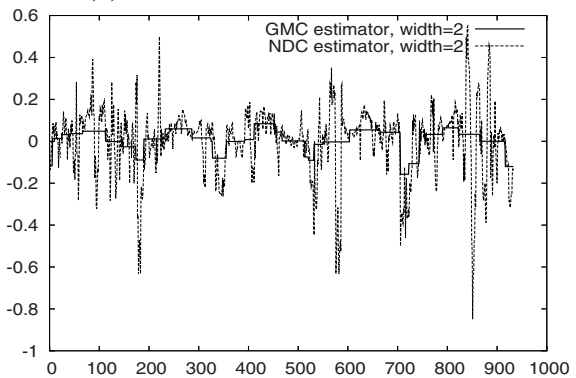

(h) "noisy" flower of Fig. 6d

Fig. 7. Experimentations on regular (a-d) and damaged (e-h) contours. Plot (a) (resp. (c)) shows the curvature precision obtained on a quarter of circle $\mathrm{R}=20$ (resp. flower) with several grid steps. Plots (b) and (d) show the results obtained by the osculating circle estimator. Curvature estimation precision on damaged shapes is shown on (e) and (g). A comparison between the GMC and NDC estimators is shown in (f,h). 
shapes with the previously described degradation algorithm. Despite a few curvature oscillations with width $\nu=1.5$, plot (e) shows that the curvature is closest to the curvature associated to the initial circle. In the same way, the curvature estimation on the flower gives correct results without concave/convex error from width 2 . Furthermore, despite the strong contour degradation, the resulting curvature field obtained with width $\nu=2$ and 3 appears stable (g), and curvature extrema are well located.

Nguyen and Debled 23] proposed a method to estimate curvature by using osculating circles and maximal blurred segments (called NDC). Here we compare this method with our approach based on tangential cover and optimization. In the two methods, the comparison was obtained by using the same blurred maximal segment algorithm as described in section 4 As shown in Fig.7f, the curvature obtained by our method is closest to the constant curvature value associated to the initial circle without noise. Contrary to the circle osculating based method, the curvature sign does not change (except for some values obtained with $\nu=1.5$ ). With the NDC method a width of $\nu=5$ was needed to obtain a constant sign value of the curvature on this contour. The comparison applied to the flower contour (Fig.7 7 -f) gives the same results. With the NDC approach, more oscillations of curvature are visible and some curvature sign inversions are still present ${ }^{4}$, even with width $\nu=3$.

The following tabular shows timing measures with the number of iterations needed by the optimization process. It was obtained with different values for the convergence criteria $(\epsilon)$. This measure was done on

\begin{tabular}{c|c|c|c}
$\epsilon$ & $\#$ iter & $E_{\max }$ & time $(\mathrm{s})$ \\
\hline $10 \mathrm{e}-4$ & 1648 & $1,38 \mathrm{e}-3$ & 0,284 \\
$10 \mathrm{e}-6$ & 7096 & $5,08 \mathrm{e}-5$ & 0,416 \\
$10 \mathrm{e}-8$ & 39101 & $5,08 \mathrm{e}-7$ & 1,028
\end{tabular}
a 2,6 GHz Intel Pentium processor with a circle of radius 1 (grid step=0.01) and with 804 surfels, 212 variables and 106 maximal segments. $E_{\max }$ represents the maximal relative error defined by $\left(\kappa_{\text {est }}^{\max }-\kappa_{\text {est }}^{\text {min }}\right) / \kappa_{\text {real }}$.

\section{Conclusion}

We have proposed a new curvature field estimator based on global minimization. In order to deal with noisy contours, this approach was extended by using blurred segments. The obtained results were compared with several recent methods and are better both on perfect and noisy contours. Future works will deal with the integration of a more efficient optimization method and the use of an optimized algorithm for blurred segment recognition. Finally, we will study the use of the tangential cover in order to automatically determine the width $\nu$ of the blurred segments.

\section{References}

1. Marji, M.: On the detection of dominant points on digital planar curves. PhD thesis, Wayne State University, Detroit, Michigan (2003)

2. Klette, R., Rosenfeld, A.: Digital Geometry - Geometric Methods for Digital Picture Analysis. Morgan Kaufmann, San Francisco (2004)

3. de Vieilleville, F., Lachaud, J.O.: Convex shapes and convergence speed of discrete tangent estimators. In: Bebis, G., Boyle, R., Parvin, B., Koracin, D., Remagnino, P., Nefian, A., Meenakshisundaram, G., Pascucci, V., Zara, J., Molineros, J., Theisel, H., Malzbender, T. (eds.) ISVC 2006. LNCS, vol. 4292, Springer, Heidelberg (2006) 
4. Lachaud, J.O.: Espaces non-euclidiens et analyse d'image : modèles déformables riemanniens et discrets, topologie et géométrie discrète. Habilitation diriger des recherches, Université Bordeaux 1, Talence, France (2006)

5. Huxley, M.N.: Exponential sums and lattice points. Proc. London Math. Soc. 60, 471-502 (1990)

6. Klette, R., Žunić, J.: Multigrid convergence of calculated features in image analysis. Journal of Mathematical Imaging and Vision 13, 173-191 (2000)

7. Kovalevsky, V., Fuchs, S.: Theoretical and experimental analysis of the accuracy of perimeter estimates. In: Förster, Ruwiedel (eds.) Proc. Robust Computer Vision, pp. 218-242 (1992)

8. Sloboda, F., Zaťko, B., Stoer, J.: On approximation of planar one-dimensional continua. In: Klette, R., Rosenfeld, A., Sloboda, F. (eds.) Advances in Digital and Computational Geometry, pp. 113-160 (1998)

9. Asano, T., Kawamura, Y., Klette, R., Obokata, K.: Minimum-length polygons in approximation sausages. In: Arcelli, C., Cordella, L.P., Sanniti di Baja, G. (eds.) IWVF 2001. LNCS, vol. 2059, pp. 103-112. Springer, Heidelberg (2001)

10. Coeurjolly, D.: Algorithmique et géométrie pour la caractérisation des courbes et des surfaces. PhD thesis, Université Lyon 2 (2002)

11. Lachaud, J.O., Vialard, A., de Vieilleville, F.: Analysis and comparative evaluation of discrete tangent estimators. In: Andrès, É., Damiand, G., Lienhardt, P. (eds.) DGCI 2005. LNCS, vol. 3429, pp. 140-251. Springer, Heidelberg (2005)

12. Lachaud, J.O., Vialard, A., de Vieilleville, F.: Fast, accurate and convergent tangent estimation on digital contours. IVC 25(10), 1572-1587 (2007)

13. Hermann, S., Klette, R.: A comparative study on 2 d curvature estimators. Research report CITR-TR-183, CITR, The University of Auckland, New Zealand (2006)

14. de Vieilleville, F., Lachaud, J.O., Feschet, F.: Maximal digital straight segments and convergence of discrete geometric estimators. JMIV 27(2), 471-502 (2007)

15. Brunet, F.: Convolutions binomiales et dérivation de fonctions discrètes bruitées. Master's thesis, LAIC. University of Clermont-Ferrand. France (2007)

16. Kass, M., Witkin, A., Terzopoulos, D.: Snakes: Active contour models. International Journal of Computer Vision 1(4), 321-331 (1988)

17. Caselles, V., Catte, F., Coll, T., Dibos, F.: A geometric model for active contours. Numerische Mathematik 66, 1-31 (1993)

18. Feschet, F., Tougne, L.: Optimal time computation of the tangent of a discrete curve: Application to the curvature. In: Bertrand, G., Couprie, M., Perroton, L. (eds.) DGCI 1999. LNCS, vol. 1568, pp. 31-40. Springer, Heidelberg (1999)

19. Debled-Rennesson, I., Feschet, F., Rouyer-Degli, J.: Optimal blurred segments decomposition of noisy shapes in linear times. Computers and Graphics (2006)

20. Buzer, L.: An elementary algorithm for digital line recognition in the general case. In: Andrès, É., Damiand, G., Lienhardt, P. (eds.) DGCI 2005. LNCS, vol. 3429, pp. 299-310. Springer, Heidelberg (2005)

21. Roussillon, T., Tougne, L., Sivignon, I.: Computation of binary objects sides number using discrete geometry application to automatic pebbles shape analysis. In: Proc. 14th International Conference on Image Analysis and Processing (2007)

22. Kanungo, T.: Document Degradation Models and a Methodology for Degradation Model Validation. PhD thesis, University of Washington (1996)

23. Nguyen, T., Debled-Rennesson, I.: Curvature estimation in noisy curves. In: Kropatsch, W.G., Kampel, M., Hanbury, A. (eds.) CAIP 2007. LNCS, vol. 4673, pp. 474-481. Springer, Heidelberg (2007) 